\title{
Études Des Caractéristiques Et Des Déterminants Des Mariages Consanguins Dans La Ville De Tiflet (Maroc)
}

\author{
Abbad Zhour \\ Drissi Ahmed \\ Abdelmajid Soulaymani \\ Khadmaoui Abderrazak
}

Département de Chimie et Biochimie, Université de Québec à Montréal, Succ. Centre-Ville, Montréal, Québec, Canada

\section{Oukarroum Abdallah}

Laboratoire de Génétique et Biométrie, Département de Biologie,

Faculté des Sciences, Université Ibn Tofail, Kénitra, Maroc

\begin{abstract}
The practice of consanguineous marriage is still very widespread in Morocco and in the Arab-Muslim world, where the customs as well as the cultural, economic and social motivations have most often an influence on the marital choice within the family. The purpose of this study is to define the determinants of this practice in the city of Tiflet and regions (Morocco). A survey was conducted on 1000 pairs randomly sampled between June and November of 2012. The results reveal a high level of consanguinity (38.9\%) and a significant association between this marital practice and the geographical and sociocultural factors such as: the place of residence before marriage, the education level, the profession and the age in the marriage.
\end{abstract}

Keywords: Consanguineous marriage, determinants, choice of spouse, Morocco, Tiflet

\section{Résumé}

La pratique du mariage consanguin reste encore très répandue au Maroc comme dans le monde arabo-musulman où les coutumes et les motivations d'ordre culturel, économique et social ont le plus souvent une influence sur le choix matrimonial à l'intérieur de la famille. Le but de cette étude est de définir les facteurs déterminants de cette pratique dans la ville de Tiflet et ses régions (Maroc). Une enquête a été menée sur 1000 couples 
échantillonnés au hasard entre juin et novembre de l'année 2012. Les résultats révèlent un niveau de consanguinité élevé (38.9\%) et une association significative entre cette pratique matrimoniale et les facteurs géographiques et socio-culturels tels que : le lieu de résidence avant mariage, le niveau d'instruction, la profession et l'âge au mariage.

Mots clés : Mariage consanguin, déterminants, choix du conjoint, Maroc, Tiflet

\section{Introduction}

Le terme de consanguinité dérive du latin : " cum » qui veut dire " avec » et " sanguins " sang ou bien plus précisément il signifie du même sang (Emery, 1986). En 1948, Malecot a introduit la notion d'identité des gènes et a développé l'approche probabiliste qui est aujourd'hui retenue pour définir et calculer les coefficients de parenté et de consanguinité.

Le mariage est dit consanguin lorsque les conjoints ont un ou plusieurs ancêtres communs. Les mariages peuvent avoir des origines géographiques ainsi à l'intérieur des petites tribus ou villages isolés ; les conjoints ont de bonnes chances de posséder des ancêtres communs (Malecot, 1948). C'est ce qu'on appelle consanguinité de position. Sous l'angle de la génétique, ceci signifie que ces deux personnes ont une probabilité non nulle de porter des gènes identiques reçus de cet ancêtre commun (Vézina et al., 2004).

L'endogamie familiale ou la consanguinité est, en effet, un cas particulier des liens matrimoniaux entres les conjoints (Valls, 1982). Les populations Arabo-musulmanes sont les plus concernées par cette pratique que d'autres (Talbi et al., 2007). L'union avec la cousine parallèle patrilatérale constitue la première forme d'endogamie familiale possible (Boua-Assy et al., 2003).

Notre enquête sur la population de la ville de Tiflet a pour objectif de déterminer le degré du mariage consanguin et les critères géographiques, culturels et socio-professionnels qui régissent cette pratique.

\section{Matériels et méthodes}

Une enquête prospective a été réalisée dans la ville de Tiflet (nordouest du Maroc), entre juin et novembre 2012, sur un échantillon de 1000 couples choisis aléatoirement. Cette enquête a été faite à l'aide d'un questionnaire, établi par le laboratoire de Génétique et Biométrie de la Faculté des Sciences de Kénitra, comprenant des questions relatives aux caractéristiques démographiques (âge, sexe, lieu de résidence,...), socioéconomiques (niveau d'instruction, profession des répondants ainsi de leurs 
parents,...) et anthropologiques (lien de parenté) des couples étudiés et de leurs parents maternels et paternels.

\section{Traitement des données}

Pour analyser l'impact des différents facteurs étudiés sur le choix du mariage consanguin chez les couples étudiés et leurs parents, nous avons fait appel au test statistique de corrélation de Pearson (Khi2 et r). Les données ont été traitées par les logiciels statistiques, Excel 2013, Statistica version 10 et IBM SPSS statistics 22.

Et pour estimer le coefficient moyen de consanguinité $(\mathrm{Ca})$, nous avons eu recours à la méthode de Jacobi et Jacquard (1971) selon laquelle ce coefficient de consanguinité $\mathrm{Ca}=\sum \mathrm{f}_{\mathrm{i}} \mathrm{F}_{\mathrm{i}}$ où fi est la fréquence relative des individus ayant le coefficient de consanguinité $\mathrm{F}_{\mathrm{i}}$.

Le coefficient de consanguinité d'un individu I se mesure à partir de la probabilité $F_{1}$ pour que les deux gènes qu'il possède, à un locus donné, soient identiques par descendance (Malécot 1948 ; jacquard 1968), soit $\mathrm{F}_{1}=\Sigma(1 / 2)^{\mathrm{np}+\mathrm{nm}+1} \mathrm{x}\left[1+\mathrm{F}_{\mathrm{A}}\right]$ où $\mathrm{n}_{\mathrm{p}}$ désigne le nombre de générations qui séparent le père de l'individu $\mathrm{I}$ de l'ancêtre $\mathrm{A} ; \mathrm{n}_{\mathrm{m}}$ désigne le nombre de générations qui séparent la mère de l'individu I de l'ancêtre $\mathbf{A}$ et $F_{A}$ désigne le coefficient de consanguinité de l'ancêtre $\mathbf{A}$.

Rappel de quelques définitions (Barry et al 2000).

Cousins germains (CG) : l'ensemble des enfants des frères et des sœurs. on distingue quatre types :

$>\quad$ Cousins parallèles : les enfants de deux frères ou de deux sœurs.

- Cousins Parallèles Patrilatéraux FFP : les enfants du frère de son père (Type I).

- Cousins Parallèles Matrilatéraux FSM : les enfants de la sœur de sa mère (Type II).

$>\quad$ Cousins croisés : les enfants de germains de sexe opposé.

- Cousins Croisés Patrilatéraux FSP : les enfants de la sœur de son père (Type III).

Cousins Croisés Matrilatéraux FFM : les enfants du frère de sa mère (Type IV).

\section{Résultats et discussion}

\section{Analyse du degré et de la nature des mariages consanguins.}

Le tableau 1 résume les mesures de consanguinité relevées dans la génération des couples étudiés et celle de leurs parents. D’après les résultats de ce tableau, nous observons que le pourcentage de consanguinité n'est que $8 \%$ chez les parents des femmes et $18 \%$ chez les parents des maris, alors que, dans la génération des couples étudiés, la consanguinité atteint un taux de $38,9 \%$, soit presque deux couples sur cinq ont tendance à s'engager dans un 
mariage consanguin. Ceci témoigne que nous sommes bien devant une région dans laquelle la pratique de la consanguinité a augmenté en passant de la génération des parents à la génération des couples étudiés.

Tableau 1 : Degré de parenté et répartition des différents types d'unions consanguines.

\begin{tabular}{|c|c|c|c|c|c|c|c|c|c|c|}
\hline \multirow{2}{*}{\multicolumn{2}{|c|}{ Générations }} & \multirow{3}{*}{$\begin{array}{l}\mathrm{CC} \\
389 \\
\end{array}$} & \multirow[b]{2}{*}{$\mathrm{CG}$} & \multirow[b]{2}{*}{$\mathrm{CI}$} & \multirow[b]{2}{*}{$\mathrm{CIG}$} & \multicolumn{4}{|c|}{$\mathrm{CG}$} & \multirow[t]{2}{*}{$\mathrm{Ca}\left(10^{-5}\right)$} \\
\hline & & & & & & \multirow{2}{*}{$\begin{array}{c}\text { FFM } \\
10 \\
\end{array}$} & \multirow{2}{*}{$\begin{array}{c}\text { FFP } \\
186\end{array}$} & \multirow{2}{*}{$\begin{array}{c}\text { FSM } \\
127\end{array}$} & \multirow{2}{*}{$\frac{\text { FSP }}{8}$} & \\
\hline GCE & ni & & 331 & 23 & 35 & & & & & 5645,5 \\
\hline & $\%$ & 38.9 & 85.1 & 5.9 & 9 & 3.02 & 56.19 & 38.37 & 2.42 & \\
\hline \multirow[t]{2}{*}{ GPM } & ni & 180 & 135 & - & 45 & 12 & 120 & 3 & - & \multirow[t]{2}{*}{5079,0} \\
\hline & $\%$ & 18 & 75 & - & 25 & 8.90 & 88.9 & 2.2 & - & \\
\hline \multirow[t]{2}{*}{ GPF } & ni & 80 & 62 & - & 18 & 1 & 60 & 1 & - & \multirow[t]{2}{*}{5195,6} \\
\hline & $\%$ & 8 & 77.5 & - & 22.5 & 1.6 & 96.8 & 1.6 & - & \\
\hline
\end{tabular}

GCE : Génération des couples étudiés ; GPM : génération des parents des maris ; GPF : génération des parents des femmes ; CC : consanguinité, CI : cousins inégaux ; CIG : cousins issus de germains ; CG : cousins germains ; FFP : Fille du frère du père ; FSP : Fille de la sœur du père ; FFM : Fille du frère de la mère ; FSM : Fille de la sœur de la mère ; Ca :coefficient de consanguinité exprimé en 10${ }^{5}$; ni : effectif dans la classe.

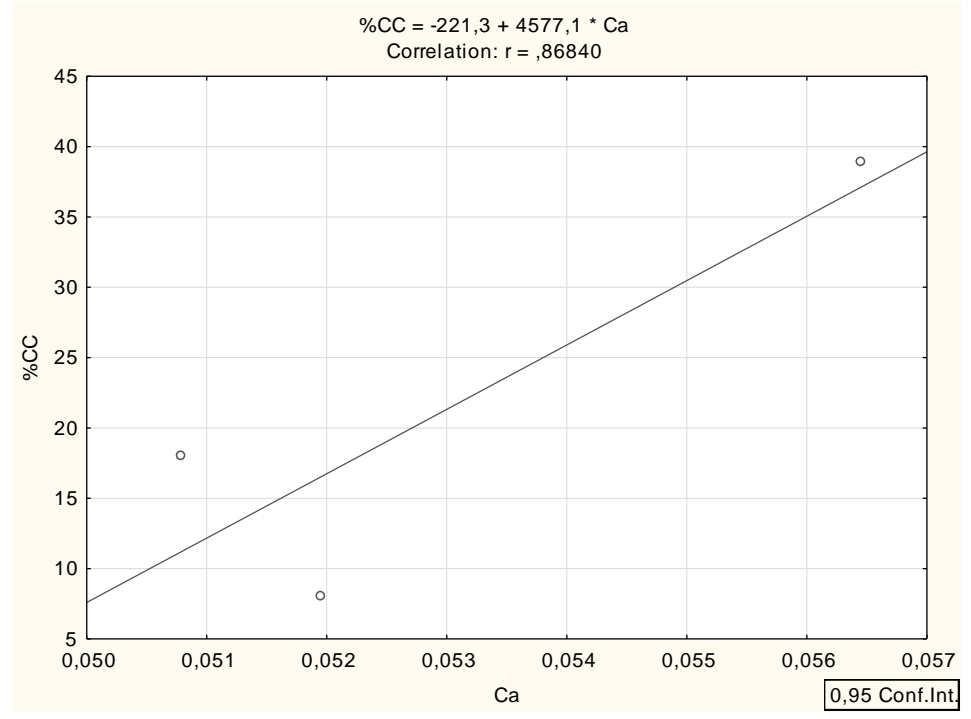

$\mathrm{Ca}$ : coefficient de consanguinité ; \%CC : pourcentage de consanguinité.

Figure 1 : Corrélation entre le pourcentage de consanguinité et le coefficient moyen de consanguinité apparente $; \mathrm{p}=0.330>0.005$.

Des études sociologiques ont confirmé que les mariages consanguins sont considérés comme importants dans le maintien de la stabilité sociale ; le couple a plus de chances d'être compatible " comme mari et femme » (Hamamy et al., 2005).

Selon l'étude de (Bou-assy et al., 2003), la consanguinité garantit une continuité de la manière d'être et de la façon de faire, une protection de l'honneur de la femme, une stabilité de la vie conjugale et une meilleure dynamique familiale, de même qu'une sécurité affective, psychologique et financière. 
Nos résultats obtenus chez les couples enquêtés concordent relativement avec ceux déclarés par Baali (1994) qui a trouvé que les unions consanguines sont fort répandues dans la Vallée d'Azgour (Haut atlas, Maroc), puisque $35.7 \%$ des couples de cette région sont impliqués dans ce type de mariage. Le même auteur a également signalé que plus la génération est ancienne plus la fréquence des unions consanguines décroît. En effet, lors de son étude, il a montré que le pourcentage de consanguinité a augmenté de $10.9 \%$ en 1904 à $56.1 \%$ dans la période 1965-1985.

Nous avons comparé également le pourcentage de la consanguinité des couples interviewés à ceux cités par plusieurs auteurs du pays voisin, l'Algérie, il en ressort que le taux de consanguinité dans notre échantillon reste supérieur à ceux déclaré par Sidi-Yakhlef, A. et al., (2013) à Oulhaça dans l'Ouest algérien (36,07\%), Moussouni A. et al., (2011) à Sabra dans le Nord-ouset algérien (33.33\%) et par Mortad N. et al., (2014) à Msirda dans l'extrême ouest algérien (30.85\%).

Quant aux coefficients de consanguinité, ils sont partiellement homogènes chez les deux générations. En effet, le coefficient de consanguinité est de 0.05645 chez la génération des couples étudiés, de $0.05079 \mathrm{chez}$ les parents des maris et de $0.05195 \mathrm{chez}$ les parents des femmes. Toutefois, cette distribution ne justifie pas celle des pourcentages de consanguinité qui témoigne d'une dissemblance plus discriminante entre ces générations (tableau 1).

Pour évaluer la concordance de la distribution des coefficients moyens de consanguinité avec celle des pourcentages de consanguinité, nous avons eu recours à la corrélation entre ces deux paramètres. La figure 1 indique que la corrélation entre les deux paramètres n'est pas significative $(\mathrm{r}=0.848 ; \mathrm{p}=0.330)$ et, par conséquent, les deux variables évoluent indépendamment.

En dépit de cette indépendance entre les deux paramètres, les coefficients de consanguinité restent élevés chez les deux générations (couples étudiés et leurs parents), cela veut dire que la proportion des loci contenant des gènes identiques est très élevée chez les descendants consanguins.

Bittles (2001) a défini, dans la génétique clinique, un mariage consanguin comme une union entre deux individus qui sont liés comme des cousins issus de germains ou plus près, avec le coefficient de consanguinité (F) égale ou supérieur à 0.0156 où $(\mathrm{F})$ représente une mesure de la proportion des loci dans lesquels la progéniture d'une union consanguine devrait hériter des copies de gènes identiques des deux parents. Dans certaines communautés, les plus hauts coefficients de consanguinité sont atteints avec les unions entre deux cousins germains pratiquées par les 
Arabes et les unions entre oncle-nièce pratiqués dans le sud de l'Inde où $(\mathrm{F})$ atteint 0,125 (Hamamy et al., 2011).

Nos résultats concernant le taux et le coefficient de consanguinité chez les couples étudiés et leurs parents ne s'accordent pas avec ceux signalés, dans différentes régions marocaines, par certains auteurs tels que Hami et al., $2009[(\operatorname{GCE}(20 \%, 0.01028), \operatorname{GMP}(17.4 \% ; 0.01025)$ et GPF (24\%; 0.01399)] dans la région de Rabat-Salé-Zemmour-Zaer (Maroc), Talbi et al., 2007 [(GCE(25.43\%; 0.0094), GPM(21.99 \%;0.0092) et GPF(20.96\%; 0.009)] dans la population marocaine et Attazagharti N.,2006 [GCE :24.8\%; 0.0166, GPM (32\%; 0.0229) et GPF (30.4\%; 0.0175$)$ ] dans la région du Gharb au Maroc.

Par ailleurs, chez la génération des couples étudiés, les mariages consanguins entre les cousins germains sont majoritairement optés et représentent $85.09 \%$, tandis que les autres types d'unions consanguines ne représentent que $14.91 \%$ (5.92\% pour les cousins inégaux et $8.99 \%$ pour les cousins issus de germains). Quant à la génération des parents, nous constatons que, chez les parents des maris comme chez les parents des femmes, trois couples sur quatre sont des cousins germains et un couple sur quatre est formé de cousins issus de germains (Tableau 1).

En outre, chez les couples étudiés, l'analyse de la répartition des alliances entre cousins germains montre que plus que la moitié des mariages (56.19\%) sont contractés avec la cousine patrilatérale parallèle (bent alaam), fille de l'oncle paternel. Cependant, la fréquence des mariages avec la cousine matrilatérale parallèle est de 38.37\%. A l'encontre, les mariages avec la cousine patrilatérale croisée ne représentent que $3.02 \%$ et les unions avec la cousine matrilatérale croisée restent manifestement les plus faibles $2.42 \%$ (Tableau 1).

Chez la génération des parents, le mariage entre cousins germains représente $75 \%$ chez la génération des parents des maris et $77.5 \%$ chez la génération des parents des femmes, ce type de mariage est le plus pratiqué dans cette génération et se fait particulièrement avec la cousine patrilatérale parallèle. En effet, la quasi-totalité des parents des femmes optent ce type d'union (96.8\%). Ainsi que, chez les parents des maris, ce type d'union reste très important $(88.9 \%)$ (Tableau 1$)$.

Pour la génération des parents des maris, les unions avec la cousine matrilatérale croisée ou la cousine matrilatérale parallèle représentent respectivement $8.9 \%$ et $2.2 \%$. Quant à la génération des parents des femmes, ces deux derniers types d'unions ne présentent que $1.6 \%$. Il est à noter que la pratique du mariage entre les cousins inégaux et le mariage avec la cousine patrilatérale croisée sont totalement absents dans la génération des parents (Tableau 1). 
En réalité, dans les sociétés arabes, toutes les catégories de cousins s'unissent entre elles (Conte, 1987), bien que le mariage entre les cousins parallèles patrilinéaires soit reconnu par le « mariage arabe ».

De nombreuses sociétés traditionnelles ont un type de mariage dit « préférentiel », notamment entre cousins. Ces mariages laissent le choix, ils n'ont pas forcément un caractère contraignant ou obligatoire, comme le mariage prescriptif, mais ils sont culturellement valorisés. La multiplication des alliances entre cousins renforce les liens de consanguinité et concentre l'héritage de la même lignée. Elle permet également d'éviter le partage des terres (Ghasarian, 1996).

Dans certaines sociétés, des écarts s'affirment entre les cousins, les patrilatéraux sont valorisés en raison de la hiérarchie sexuelle. Ces écarts varient en fonction du type de mariage réalisé par les parents : si la sœur du père a épousé un agnat, il est certain que ses enfants entretiendront avec les membres de la fratrie des liens fondés sur une identité agnatique renforcée, ce qui ne sera pas le cas si elle se marie en dehors du groupe agnatique (Lévi-Strauss, 1976).

En somme, Malgré la régression que présentent les mariages entre cousins germains au fil des générations, leurs fréquences restent encore très élevées dans notre population avec une dominance du mariage avec la cousine patrilatérale parallèle. Cette tradition a été rencontrée par Talbi et al. en 2007 dans la population marocaine et par Hami et al. en 2005dans la population de Kenitra. Ces résultats concordent également avec les données rapportées par les Enquêtes Démographiques et de la Santé menées au Maroc (ENPS-I 1987. ENPS-II 1992).

\section{Les facteurs déterminants du mariage consanguin. Lieu de résidence avant mariage.}

Pour avoir une idée sur les déterminants intervenant dans la pratique des mariages consanguins dans la ville de Tiflet, nous avons établi un ensemble de croisements entre les statuts des conjoints consanguins (le lieu de résidence avant mariage, la profession, le niveau d'instruction et l'âge du mariage) dans notre population totale.

D'autres investigations ont cherché à expliquer ce choix matrimonial dans certains pays et, en conséquence, elles ont avancé qu'un grand nombre de facteurs liés aux caractéristiques des femmes, pourraient être des déterminants du choix de ce type de mariage, à savoir le niveau d'instruction (Al Husain et Al Bunyan, 1997; Hussain, 1998; Alper et al., 2004; Hami et al. 2009), le milieu de socialisation (Hussain et Bittles, 1998; Hami et al. 2009), la profession (Khlat, 1988; Jurdi et Saxena, 2003; Hami et al., 2009) et l'âge d'entrée en première union (Afzal et al., 1994; Hussain et Bittles, 1999; Gunaid et al., 2004; Hami et al., 2009 ). 


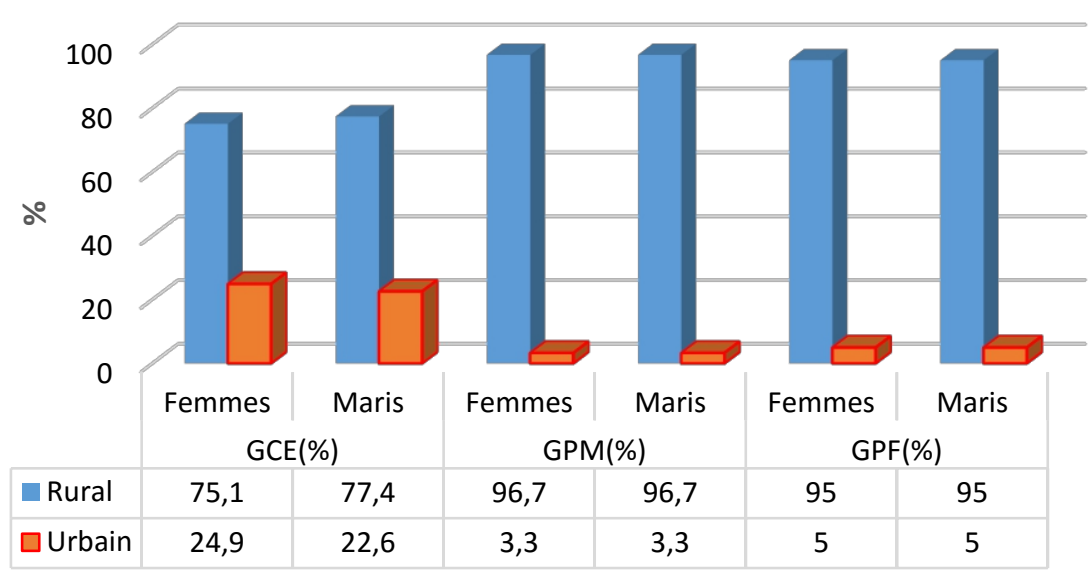

GCE: Génération des couples étudiés; GPM: Génération des parents des maris; GPF: Génération des parents des femmes.

Rural $\square$ Urbain

Figure 2 : Répartition des couples consanguins selon lieu de résidence avant mariage dans les deux générations.

L'analyse statistique des données relatives à la distribution des couples consanguins, selon lieu de résidence avant mariage, révèle que le pourcentage des individus, ayant vécu dans le milieu rural avant le mariage, est très élevé chez les femmes comme chez les hommes dans les deux générations. Toutefois, ce pourcentage a connu une diminution en passant de la génération des parents $(96,7 \%$ chez les parents des maris et $95 \%$ chez les parents des femmes) à la génération des couples étudiés (76.25\%) (figure 2).

Dans le but de vérifier l'existence d'une relation entre le lien de parenté des conjoints et le milieu de résidence avant mariage, nous avons estimé la corrélation entre le milieu de résidence avant le mariage de la femme et celui de son conjoint, et ce, dans les deux générations. Ensuite, nous avons procédé au calcul des coefficients de corrélation de Pearson afin d'évaluer la signification de cette corrélation.

Tableau 2 : Tableau croisé de lieu de résidence avant mariage des couples consanguins dans les deux générations.

\begin{tabular}{|c|c|c|c|}
\hline \multirow{2}{*}{\multicolumn{2}{|c|}{ Génération des couples étudiés }} & \multicolumn{2}{|c|}{ Femmes } \\
\hline & & \multirow{2}{*}{$\begin{array}{c}\text { Milieu rural (\%) } \\
\mathbf{7 1 . 9 8}\end{array}$} & \multirow{2}{*}{$\begin{array}{c}\text { Milieu urbain }(\%) \\
\mathbf{5 . 4 0}\end{array}$} \\
\hline & Milieu rural $(\%)$ & & \\
\hline Maris & Milieu urbain (\%) & 3.08 & 19.54 \\
\hline \multicolumn{4}{|c|}{ Effectif total $(N)=389$, Khi2 $=229,267, P=0,000 ; d d l=1$} \\
\hline \multicolumn{4}{|c|}{ Génération des parents des maris } \\
\hline \multirow[t]{2}{*}{ Maris } & Milieu rural (\%) & 96.67 & - \\
\hline & Milieu urbain (\%) & - & 3.33 \\
\hline \multicolumn{4}{|c|}{ Effectif total $(N)=180$, Khi2 $=180,000, p=0,000 ; d d l=1$} \\
\hline \multicolumn{4}{|c|}{ Génération des parents des femmes } \\
\hline \multirow[t]{2}{*}{ Maris } & Milieu rural (\%) & 95 & - \\
\hline & Milieu urbain (\%) & - & 5 \\
\hline \multicolumn{4}{|c|}{ Effectif total $(N)=80 ;$ Khi2 $=80,000 ; p=0,000 ; d d l=1$} \\
\hline
\end{tabular}


A la lumière des résultats du tableau 2, nous avons observé une dominance des mariages entre les conjoints ayant vécu dans le milieu rural avant leur mariage. En effet, le taux des couples étudiés consanguins, ayant résidé dans le milieu rural avant leur mariage, atteint plus de $70 \%$.

Quant à la génération des parents consanguins, ce taux y dépasse 95\%. Par conséquent, le taux des mariages entre les individus qui ont vécu dans le milieu rural avant leur mariage a diminué en passant de la génération des parents à la génération des couples étudiés.

En dépit de cette diminution, Le test statistique Khi2 indique que la résidence dans le milieu rural avant mariage a un effet hautement significatif sur le choix du mariage consanguin chez les deux générations (chez les couples étudiés Khi2 $=229,267 ; \mathrm{p}=0,000$, chez les parents des maris Khi2 $=180,000 ; p=0,000$ et chez les parents des femmes Khi2 $=80,000$; $\mathrm{p}=0,000)$.

Nos résultats concordent avec ceux cités par Chalbi (2009) qui a rapporté que les pourcentages de consanguinité sont élevés en milieu rural où la propriété de terrains agricoles et le travail de la terre sont déterminants.

Des études menées en Inde (Dronamaraju et Khan, 1962-1963; Rao et Inbaraj, 1979), en Egypte (Hafez et al., 1983), au sud du Brésil (FreireMaia et al., 1983) et au Maroc (Talbi, 2002; Hami, 2007) ont montré également que le niveau de consanguinité est plus élevé dans le milieu rural.

La survivance de cette tradition, dans les nouvelles générations avec autant de force comme ils le faisaient les générations les plus âgées en particulier dans les zones rurales (Ghazi et al., 2009), trouve son origine dans la variabilité de ses motivations qui sont en général d'ordre : social, économique, culturel, religieux, géographique et politique.

\section{Niveau d'instruction.}

\begin{tabular}{|c|c|c|c|c|c|c|}
\hline$\frac{\grave{a}}{a}$ & $\begin{array}{r}\text { Femmes } \\
\text { GCE }\end{array}$ & $\begin{array}{l}\text { Maris } \\
\%)\end{array}$ & $\begin{array}{r}\text { Femmes } \\
\text { GPM }\end{array}$ & $\begin{array}{l}\text { Maris } \\
\text { (\%) }\end{array}$ & $\begin{array}{r}\text { Femmes } \\
\text { GPF }\end{array}$ & $\begin{array}{l}\text { Maris } \\
\%)\end{array}$ \\
\hline 돈 Analphabète & 63,8 & 42,4 & 98,9 & 95 & 100 & 93,8 \\
\hline 气̆ Primaire & 15,9 & 25,7 & 1,1 & 5 & 0 & 6,3 \\
\hline 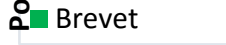 & 9,8 & 14,7 & 0 & 0 & 0 & 0 \\
\hline Baccalauréat & 7,7 & 11,8 & 0 & 0 & 0 & 0 \\
\hline Supérieur & 2,8 & 5,4 & 0 & 0 & 0 & 0 \\
\hline
\end{tabular}

Figure 3 : Répartition des couples consanguins selon le niveau d'instruction dans les deux générations. 
D'après les résultats concernant la répartition des couples consanguins, selon le niveau d'instruction, dans la ville de Tiflet et ses régions, nous avons constaté que le taux d'analphabétisme est très élevé chez la génération des couples enquêtés comme chez leurs parents. En effet, les maris des couples interviewés non instruits représentent la grande part (42.4\%) par rapport aux autres catégories du niveau d'instruction. Les maris ayant le niveau primaire viennent en deuxième rang avec un pourcentage de $25 \%$. De même, chez les femmes des couples étudiés, nous avons noté la dominance des deux statuts «analphabète» et "primaire» et qui représentent respectivement $63.8 \%$ et $15,9 \%$. Quant à la génération des parents, la majorité d'entre eux sont illettrés. En effet, les parents des maris non scolarisés représentent $95 \%$ pour les pères et $8.9 \%$ pour les mères. Même constatation a été faite chez les parents des femmes qui représentent un taux très élevé d'analphabétisme, soit $93.7 \%$ pour les pères et $100 \% \mathrm{chez}$ les mères (figure 3 ).

Tableau 3 : Tableau croisé des niveaux d'instruction des couples consanguins dans les deux générations.

\begin{tabular}{|c|c|c|c|c|c|c|}
\hline \multicolumn{7}{|c|}{ Génération des couples étudiés } \\
\hline & \multicolumn{6}{|c|}{ Femmes } \\
\hline & & Analphabète(\%) & Primaire(\%) & Brevet $(\%)$ & Baccalauréat(\%) & Supérieur(\%) \\
\hline \multirow{5}{*}{ Maris } & $\begin{array}{c}\text { Analphabète } \\
(\%)\end{array}$ & 37.79 & 2.83 & 1.80 & - & - \\
\hline & $\begin{array}{c}\text { Primaire } \\
(\%)\end{array}$ & 16.71 & 7.18 & 1.29 & 0.51 & - \\
\hline & Brevet $(\%)$ & 5.91 & 2.57 & 3.34 & 2.57 & 0.26 \\
\hline & $\begin{array}{c}\text { Baccalauréat } \\
(\%)\end{array}$ & 3.08 & 2.83 & 1.80 & 3.86 & 0.26 \\
\hline & $\begin{array}{c}\text { Supérieur } \\
(\%)\end{array}$ & 0.26 & 0.51 & 1.54 & 0.77 & 9.31 \\
\hline \multicolumn{7}{|c|}{$\mathrm{N}=389 ;$ Khi2 $=279,177 ; \mathrm{p}=0.000 ; \mathrm{ddl}=16$; Taux d'homogamie calculé $: 61,48 \%$} \\
\hline \multicolumn{7}{|c|}{ Génération des parents des maris } \\
\hline & \multicolumn{6}{|c|}{ Femmes } \\
\hline & & \multicolumn{2}{|c|}{ Non instruites $(\%)$} & \multicolumn{3}{|c|}{ Instruites (\%) } \\
\hline \multirow[t]{2}{*}{ Maris } & $\begin{array}{c}\text { Non } \\
\text { instruits }(\%)\end{array}$ & 94.44 & & \multicolumn{3}{|c|}{0.56} \\
\hline & Instruits $(\%)$ & 4.44 & & & 56 & \\
\hline \multicolumn{7}{|c|}{$\mathrm{N}=180 ;$ Khi2 $=8,622 ; \mathrm{p}=0.003 ; \mathrm{ddl}=1$} \\
\hline \multicolumn{7}{|c|}{ Génération des parents des femmes } \\
\hline \multirow[t]{2}{*}{ Maris } & $\begin{array}{c}\text { Non } \\
\text { instruits }(\%)\end{array}$ & 93.75 & & \multicolumn{3}{|c|}{ - } \\
\hline & Instruits(\%) & 6.25 & & & - & \\
\hline
\end{tabular}

Les résultats du tableau 3 indiquent que les conjoints consanguins des couples étudiés se choisissent de même niveau d'étude à $61.48 \%$ et la grande part d'homogamie éducationnelle revient aux couples analphabètes qui représentent $37.79 \%$. Quant à la génération des parents, nous avons constaté que $94.09 \%$ des couples consanguins sont non instruits $(94,44 \%$ chez la 
génération des parents des maris et $93.75 \%$ chez la génération des parents des femmes).

L'étude des résultats dans notre population indique que le pourcentage des femmes et des maris consanguins et analphabètes est plus élevé par rapport aux autres catégories, et ce, chez la génération des couples étudiées comme chez la génération de leurs parents. En outre, $37.79 \%$ des maris analphabètes, dans la génération des couples étudiés, préfèrent se marier avec des femmes de même statut culturel. Quant à la génération des parents, nous avons constaté que $94.09 \%$ des couples consanguins sont non instruits $(94.44 \%$ chez la génération des parents des maris et $93.75 \%$ chez la génération des parents des femmes).

Le test statistique Khi2 indique que le niveau d'instruction de la catégorie "analphabète » ou "non instruit» a un effet significatif sur le choix du mariage consanguin chez les deux générations (chez les couples étudies Khi2 $=279,177 ; p=0.000$ et chez la génération des parents des maris Khi2 $=8,622 \mathrm{a} ; \mathrm{p}=0.003$ ).

Nos résultats sont en harmonie avec ceux obtenus à Oulhaça dans l'Ouest algérien par Sidi-Yakhlef, A. et al., (2012) qui a déclaré qu'une femme ayant un niveau bas d'étude (analphabète ou primaire) a de forte chance d'être en union consanguine qu'une femme ayant le niveau supérieur. Nos résultats sont également similaires à ceux obtenus par d'autres recherches (Khoury et Massad, 1992; Benhamadi, 1997; Jurdi et Saxena, 2003; Barbou et Salameh, 2009).

Dans certains pays arabes, lorsque le niveau d'éducation est élevé chez les femmes, le niveau de consanguinité baisse (Khoury et Massad, 1992). Contrairement, dans certaines sociétés, lorsque le niveau d'éducation est élevé chez les hommes, ceux -ci préfèrent se marier avec leurs cousines (Jurdi et saxena, 2003).

\section{Profession}

La lecture des données du tableau 4, concernant la répartition des professions chez la population totale, montre que, chez les couples interrogés, il y a une dominance des maris consanguins ayant un statut " profession intermédiaire » ou « agriculteur exploitant» et qui représentent respectivement $32.1 \%$ et $28.8 \%$. Quant aux pères consanguins des maris et des femmes, presque les trois quarts d'entre eux sont des agriculteurs exploitants. Tandis que, chez les femmes consanguines de notre population, la majorité est « sans activité » (96,4\% chez les femmes des couples étudiés, $100 \%$ chez les mères des maris et $80 \%$ chez les mères femmes).

En s'appuyant sur l'analyse de ces résultats, il semble que les travaux dans le domaine agricole et les professions intermédiaires sont les plus fréquents chez les maris consanguins des couples étudiés, ces derniers ont 
tendance à contracter un mariage consanguin avec des femmes sans activité. Il est à noter que les maris des couples étudiés, à l'encontre de leurs parents, se sont orientés vers d'autres catégories de profession notamment la «profession intermédiaire» tout en conservant ce comportement matrimonial.

Les résultats enregistrés lors d'une étude menée par Hami, H. et al., 2007 dans les deux régions Rabat-Salé-Zemmour-Zaer et Gharb-ChrardaBeni-Hssen ont montré que la quasi-totalité des femmes consanguines sont déclarées « sans activité ».

Tableau 4 : Répartition des couples consanguins selon le niveau d'instruction dans les deux générations.

\begin{tabular}{|c|c|c|c|c|c|c|c|}
\hline \multirow[b]{2}{*}{ Profession } & & \multicolumn{2}{|c|}{ GCE } & \multicolumn{2}{|c|}{ GPM } & \multicolumn{2}{|c|}{ GPF } \\
\hline & & Femmes & Maris & Femmes & Maris & Femmes & Maris \\
\hline \multirow[t]{2}{*}{ Ouvrier non qualifié } & $\mathbf{n}$ & - & 20 & - & 12 & - & - \\
\hline & $\%$ & - & 5 & - & 6,7 & - & - \\
\hline \multirow[t]{2}{*}{ Ouvrier qualifié } & $\mathbf{n}$ & $\mathbf{1}$ & 20 & - & 2 & - & - \\
\hline & $\%$ & 0.3 & 5,1 & - & 1,1 & - & - \\
\hline \multirow[t]{2}{*}{ Employé } & $\mathbf{n}$ & 3 & 34 & - & $\mathbf{0}$ & - & 9 \\
\hline & $\%$ & $\mathbf{0 , 8}$ & 8,7 & - & $\mathbf{0}$ & - & 11,3 \\
\hline \multirow[t]{2}{*}{ Profession intermédiaire } & $\mathbf{n}$ & 4 & 125 & - & 16 & - & 4 \\
\hline & $\%$ & 1 & 32,1 & - & 8,9 & - & 5 \\
\hline \multirow[t]{2}{*}{ Cadre supérieur } & $\mathbf{n}$ & 5 & 18 & - & - & - & - \\
\hline & $\%$ & 1,3 & 4,6 & - & - & - & - \\
\hline \multirow[t]{2}{*}{ Agriculteur exploitant } & $\mathbf{n}$ & - & 112 & - & 134 & - & 51 \\
\hline & $\%$ & - & 28,8 & - & 74,4 & - & 63,7 \\
\hline \multirow{2}{*}{$\begin{array}{c}\text { Artisan/commerçant/Chef } \\
\text { d'entreprise }\end{array}$} & $\mathbf{n}$ & 1 & 59 & - & 16 & - & 15 \\
\hline & $\%$ & $\mathbf{0 , 3}$ & 15,2 & - & 8,9 & - & 18,8 \\
\hline \multirow[t]{2}{*}{ Pas d'activité } & $\mathbf{n}$ & 375 & 1 & 180 & $\mathbf{0}$ & 80 & 1 \\
\hline & $\%$ & 96,4 & $\mathbf{0 , 3}$ & 100 & $\mathbf{0}$ & 100 & 1,3 \\
\hline \multirow[t]{2}{*}{ Total } & $\mathbf{n}$ & 389 & 389 & 180 & 180 & 80 & 80 \\
\hline & $\%$ & 100 & 100 & 100 & 100 & 100 & 100 \\
\hline
\end{tabular}

selon Audinarayana et Krishnamoorthy (2000) et Jurdi et Saxena (2003), le fait de vivre dans des conditions socio-économiques défavorables, augmente de manière significative la probabilité d'être en unions consanguines, ce qui contribue au maintien de ces pratiques traditionnelles. En fait, nombre d'auteurs soulignent l'importance de ces mariages en milieu rural, parmi les individus les plus pauvres et de faible niveau d'instruction, en fait ce type de mariage paraît comme une entre-aide entre les deux familles (Hussain, 1999; Bittles, 2001a et 2001b; Bittles, 2002). Mais aussi exceptionnellement chez les riches propriétaires. Dans ce cas, la considération la plus importante serait la préservation du patrimoine familial (Schull et Neel, 1965; Chelhod, 1965). 


\section{Age du mariage}

Les résultats de la figure 4 montrent que $35 \%$ des femmes consanguines se sont mariées dans la tranche d'âge [moins de 20 ans]. Par contre, le pourcentage des maris, qui ont contracté un mariage dans cette tranche d'âge, est très faible et ne représente que 2,6\%. De même, la fréquence des unions consanguines dans la tranche d'âge [20 ans-24ans] reste élevée chez le sexe féminin (45\%) par rapport au sexe masculin $(17,7 \%)$. A l'inverse, le pourcentage des maris qui se sont mariés dans les tranches d'âge de [25 ans et plus] est très supérieur à celui des femmes (figure 4). Ces résultats montrent que l'âge au moment du mariage est un facteur déterminant dans le choix d'un conjoint consanguin ou d'une conjointe consanguine $(\mathrm{p}=0,000, \mathrm{Khi} 2=300,088)$.

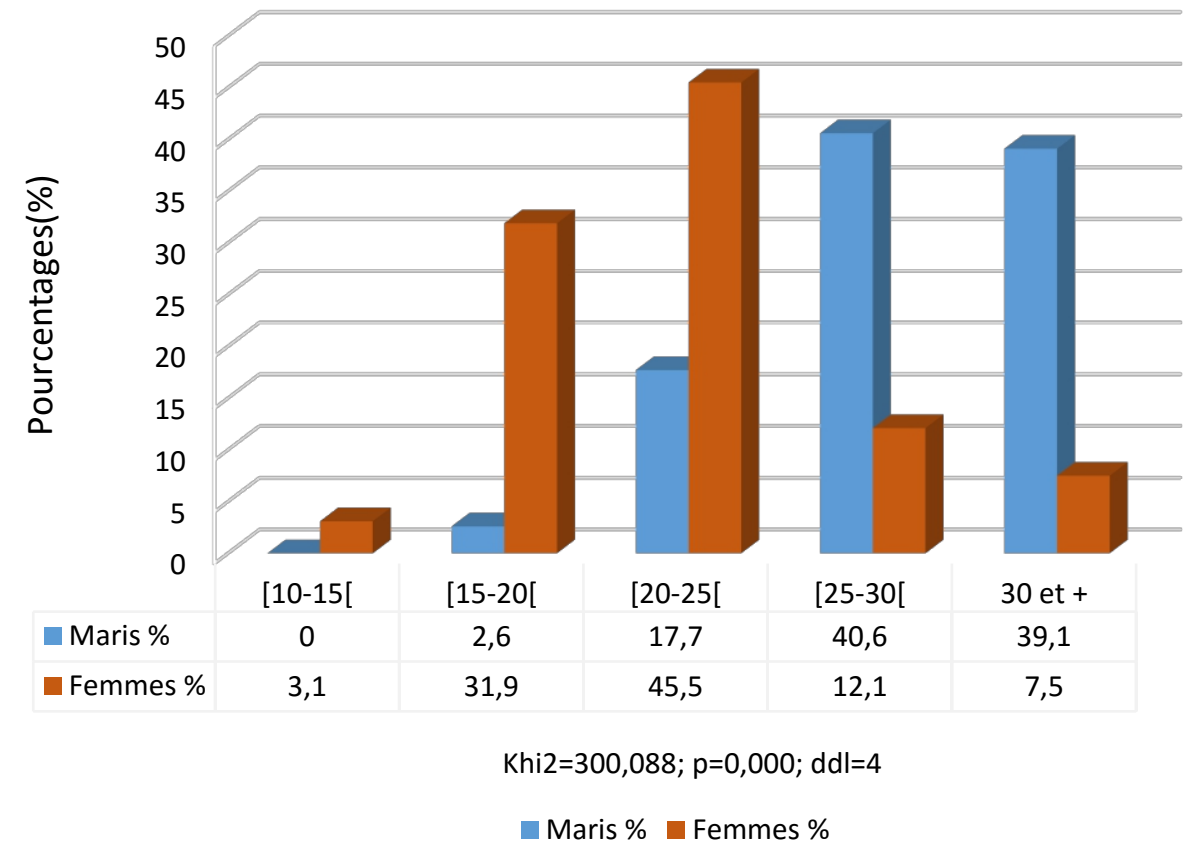

Figure 4 : Répartition des âges chez les couples étudiés et consanguins

Les résultats obtenus par Sidi-Yakhlef, A. et al., (2012) à Oulhaça dans l'Ouest algérien ont montré également que l'âge précoce des femmes $(\leq$ 18 ans) est associé à l'acceptation des unions consanguines avec $26,31 \%$. Ces résultats suggèrent ainsi, que plus les femmes se marient jeunes, plus elles sont susceptibles d'accepter cette forme de mariage. Selon Khlat (1989), la pratique des mariages consanguins à un âge précoce signifie une plus grande adhésion au modèle traditionnel et reflète essentiellement le fait que le mariage n'est pas une décision individuelle, mais a été préparé et négocié par les deux familles depuis l'adolescence des futurs époux. 


\section{Conclusion}

Dans cette étude, nous avons constaté que, d'une part, le taux de consanguinité a augmenté en passant de la génération des parents à la génération des couples étudiés qui ont renforcé cette pratique matrimoniale avec une dominance du mariage avec la cousine patrilatérale parallèle dans les deux générations. D’autre part, les coefficients de consanguinité sont élevés et partiellement homogènes chez les deux générations. C'est-à-dire la proportion des loci contenant des gènes identiques est très élevée chez les descendants consanguins.

Nous avons noté également que les coefficients moyens de consanguinité et le taux de consanguinité évoluent indépendamment dans les deux générations.

D'ailleurs, nos résultats confirment une association significative entre les facteurs étudiés (le lieu de résidence avant mariage, le statut socioprofessionnel, le niveau d'instruction et l' âge de mariage) et la pratique du mariage consanguin.

Dans les sociétés arabo-musulmanes, la consanguinité est influencée par des facteurs géographiques, démographiques, religieux, culturels et socio-économiques. Les taux les plus élevés d'unions consanguines ont été associés à un faible statut socio-économique, faible niveau d'instruction et la vie dans les zones rurales (Kerkeni et al., 2007).

\section{References:}

Afzal, M., Ali, S.M., et Siyal, H.B., 1994, Consanguineous marriages in Pakistan. Pakistan Development Review, 33 (4), 663-674.

Al Husain, M. et albunyan, M., 1997, Consanguineous marriages in a Saudi population and the effect of inbreeding on prenatal and postnatal mortality. Ann Trop Paediatr 17 (2), 155-160.

Alper O.M, Erengin H, Manguoglu A.E, Bilgen T, Cetin Z, Dedeoglu N, Luleci G., 2004. Consanguineous marriages in the province of Antalya, Turkey. Annales de Génétique, 47 (2) : 129-138.

Attazagharti N., Hami H., Soulaymania., Benali D., Khadmaouia (2006). Consanguinité et Isonymie dans la Région du Gharb au Maroc. Biologie \& Santé vol. 6, $\mathrm{n}^{\circ} 2$.

Audinarayana, N., Krishnamoorthy, S. 2000. Contribution of social and cultural factors to the decline in consanguinity in South India. Social Biology, 47, 189- 200.

Baali, A., 1994, Etude anthropobiologique d'une population berbère semiisolée du Haut-Atlas: Vallée d'Azgour, cercle d'Amizmiz, Marrakech. Thèse de Doctorat d'Etat, Faculté Sciences Semlalia, Marrakech.

Barbou, B., \& Salameh, P. (2009). Consanguinity in Lebanon: prevalence, distribution and determinants. J Biosoc Sci , 41, 505-517. 
Barry L.S, Bonte P.,D’onofrio S.,Govoroff N., Jamard J.,L., Mathieu N-C., Gené E.P., Wilgaux J., Zemplèni A., Zonabend F. Glossaire de la parenté. L'homme 2000, 154-155, 721-732.

Benhamadi, B., 1997, Les déterminants de l'endogamie au Maroc, DHS I et II. Thèse Doctorat, Université de Montréal, Canada.

Bittles A (2001) Consanguinity and its relevance to clinical genetics. Clin Genet 60:89-98

Bittles, A.H. A Background summary of consanguineous marriage. Edith Cowan University 2001a, Perth, Aystralia

Bittles, A.H. Consanguinity and its relevance to clinical genetics. Clinical Genetics 2001b, 60 (2), 89-98.

Bittles, A., (2002). Endogamy, Consanguinity and community genetics. 81 (3), 91-98.

Bou-assy F, Dumont S, Saillant F., 2003. Représentations sociales du mariage endogame et de ses conséquences biologiques sur la santé des descendants chez des fiancés apparentés : Cas de deux villages chiites au Liban. Service social, Vol 50 n¹, Pages : 174-198.

Chalbi N (2009). Les unions entre individus apparentés en Tunisie. Importance, motivations sociales et conséquences sur la mortalité préreproductive: Avant le mariage : les fiançailles dans les sociétés contemporaines. XXVI IUSSP International Population Conference, Marrakech ; 1-17.

Chelhod, J. (1965). Le mariage avec la cousine parallèle dans le système arabe. L'Homme , 7 (3-4), 113-173.

Conte E (1987). Alliance et parenté élective en Arabie ancienne. Éléments d'une problématique. In: L'Homme, tome 27 n¹02. pp. 119-138.

Doormaal et al., 2008. La consanguinité au fil du temps. Reseau laitier Canadien / Canadian dairy network, Guelph, Ontario.

Dronamaraju KR., Khan PM., 1962-1963, The frequency and effects of consanguineous marriages in Andhra Pradesh. Journal of Genetics, 58, 387401 .

Emery A.E.H, 1986. Abrégés génétique médicale. Masson, Pages 131-154.

Enquête Nationale sur la Population et la. Santé (ENPS-I). 1987

Enquête Nationale sur la Population et la. Santé (ENPS-II). 1992

Freire-Maia N., Chautard Freire-Maia EA., Aguiar-Wolter IP., et al, 1983, Inbreeding studies in Brazilian schoolchildren. I. Introduction. Materials and methods. American Journal of Medical Genetics, 16, 331-6.

Ghasarian, Christian. 1996. Introduction à l'étude de la parenté. Paris : Éditions du Seuil, p 276

Ghazi O.T, Pratibha N, Tasneem O, Mahmoud T.A.A, Najib A.K and Hanan A.H., 2009. Consanguinity and reproductive health among Arabs. Reproductive Health, 6: 17 doi:10.1186/1742-4755-6-17. 
Gunaid, A.A., Hummad, N.A., et Tamim, K.A., 2004, Consanguineous marriage in the capital city Sana'a, Yemen. Journal of Biosocial Science, 36 (1), 111-121.

Hafez, M., El-Tahan, H., Awadallah, M., El-Khayat, H., Abdelgafar, A. et Ghoneim, M., 1983, Consanguineous mating in Egyptian population. Journal of Medical Genetics 20, 58-60.

Hamamy H., Jamhawi L., Al-darawsheh J. et AJlouni K., 2005, Consanguineous marriages in Jordan: why is the rate changing with time? Clinical Genetics, 67, 511-516.

Hami H., 2007, Déterminants des mariages consanguins au Maroc: cas de deux régions du Nord-Ouest. Thèse de Doctorat, Faculté des Sciences, Kenitra.

Hami, H., Soulaymani, A., Mokhtari, A., 2009, Les Déterminants des Mariages Consanguins dans la Région de Rabat-Salé-Zemmour-Zaer (Maroc). Antropo, 18, 27-35. www.didac.ehu.es/antropo

Hussain, R., 1998, The role of consanguinity and inbreeding as a determinant of spontaneous abortion in Karachi, Pakistan. Annals of Human Genetics, 62, 147-157.

Hussain, R., et Bittles, A.H., 1998, The prevalence and demographic characteristics of consanguineous marriages in Pakistan. Journal of Biosocial Science, 30 (2), 261- 275.

Hussain, R., et Bittles, A.H., 1999, Consanguineous marriage and differentials in age at marriage, contraceptive use and fertility in Pakistan. Journal of Biosocial Science, 31(1), 121-138.

Hussain R., 1999: Community perceptions of reasons for preference for consanguineous marriages in Pakistan. Journal of Biosocial Science, 31(4), 449-461.

Jacquard A. Panmixie et consanguinité. Quelques précisions de langage. Population 1968. 6, 1065-1090.

Jakobi, L. et Jaquard, A., 1971, Consanguinité proche, consanguinité éloignée. Essai de mesure dans un village breton. INED Paris, 60, 263-268.

Jurdi, R., et Saxena, P.C., 2003, The prevalence and correlates of consanguineous marriages in Yemen: similarities and contrasts with other Arab countries. Journal of Biosocial Science, 35 (1), 1-13.

Kerkeni K , Monastiri K , Seket B , Guediche MN, Ben Cheikh H (2007). Interplay of Socio-economic Factors, Consanguinity, Fertility, and Offspring Mortality in Monastir, Tunisia Croat Med J.;48:701-7.

Khlat, M., 1988, Consanguineous marriage and reproduction in Beirut, Lebanon. American Journal of Human Genetics, 43 (2), 188-196.

Khoury SA, D. Massad (1992). Mariages consanguins dans la Jordanie. Am J Med Genet: 43 (5): 769-775.

Lévi strauss, C. (1976). Anthropologie structurale. Paris, Pocket , 480. 
Malécot G, 1948. Les mathématiques de l'hérédité. Masson, Edit .Paris.

Mortad nedjlaà et al. ( 2014) Etude Bio-anthropologique des mariages consanguins sur la morbidité et les paramètres de fitness chez la population de Msirda dans l'extrême Ouest algérien; p 19.

Moussouni, A., Aouar Metri, A (2011). Etude Anthropo- biologique de la consanguinité sur les paramètres de fitness et de morbidité dans la population de Sabra dans l'Ouest Etude comparative dans le bassin Méditerranéen. p65. Rao PSS. et Inbaraj SG., 1979, Trends in human reproductive wastage in relation to long-term practice of inbreeding. Annals of Human Genetics, 42, 401-13.

Talbi J., 2002, Consanguinité, endogamie et isonymie dans la région de Doukkal, Maroc. Mémoire de DESA, Université Chouaïb Doukkali, faculté des Sciences, El-Jadida.

Schull, W.J. and Neel, J.V., 1965: The effects of inbreeding on Japanese children. Harper and Rcow, New-York, 419 pp.

Sidi-Yakhlef, A., Aouar Metri, A., 2012. Etude Anthropo-sociologique de la consanguinité dans la population de «Oulhaça» dans l'Ouest Algérien $p$ 70,72 .

Sidi-Yakhlef, A., Aouar Metri, A., 2013. Etude Anthropo-sociologique de la consanguinité dans la population de «Oulhaça» dans l'Ouest Algérien. Antropo, 30, 45-59. www.didac.ehu.es/antropo

Talbi, J., Khadmaoui, A. E., Soulaymani, A. E. M., Chafik, A. E. A., 2007, Etude de la consanguinité dans la population marocaine. Impact sur le profil de la santé, Antropo, 15, 1-11. www.didac.ehu.es/antropo

Valls A, 1982. Anthropologia de la consanguinidad. Editorial de la Universidad Complutence, Madrid.

Vézina H, Tremblay M, Houde L., 2004. Mesures de l'apparentement biologique au Saguenay-Lac-St-Jean (Québec, Canada) à partir de reconstitutions généalogiques annales de démographie historique, $n^{\circ} 2: 67$ 84. 